\title{
New Type Intelligent Infrared Driving Bird Device
}

\author{
Zheyu Zhao \\ China, Beijing, Changping country \\ 18811300277@163.com
}

\begin{abstract}
Keywords: Infrared intelligence; Device of driving birds
\end{abstract}
\begin{abstract}
In recent years, due to the improvement of human ecological environmental protection awareness, more birds can live in harmony with mankind. Therefore, it also has a significant impact on the human production. Therefore, this paper designed a device for driving birds, in order to drive birds , which can not harm birds either. The utility model has the advantages of energy saving, environmental protection, strong adaptability and wide application range. Has great potential for development and utilization value.
\end{abstract}

\section{Background.}

Birds are human friends, which is the nature of the most intelligent one. In recent years, with the development of urban and rural ecology, and the increasing awareness of the protection of human ecology, the conditions for birds to live and reproduce are constantly improving, and the number and species of birds are increasing. Birds take us many benefits as everyone knows, but birds also normal production and operation of the high-voltage transmission line, Shaichang, orchard, airports and other forms a huge impact, causing major losses. Therefore, it is an urgent problem that how to avoid the influence of birds on the normal production and life of human beings without hurting the birds.

The Impact of Birds on Transmission Lines. With the development of the national economy, the supply of electricity has become the premise of the development of all walks of life, as an indispensable part of the normal supply of electricity, transmission line itself is very fragile. Transmission line is an important part of the transmission network, especially in the power grid, which is the key to the safe, economic and reliable operation. Due to the high voltage transmission lines are mostly distributed in the wild, it covers a wide range, the ecological environment is changeable, so it is undoubtedly subject to natural disasters and animal damage, such as unforeseen damage. In the aspects of the traditional artificial inspection workload is a major challenge of line detection, and artificial transmission line is prone to false detection and missed etc., is difficult to guarantee the accuracy of detection results, caused a great loss to the national economy.

The transmission tower bar compared to the trees, the structure is more stable, not easily affected by rain and snow storm caused violent shaking, so bird nest will be more willing to ride in the tower; in addition, some local forest trees less, birds had to perch on the power line, nesting in the tower bar. With branches into the nest encountered rainy weather, the tower of the bird's nest is blown by the wind dropped in the electric wire or hanging on the bottle, the branch contact wire (or near conductor) will short-circuit grounding accident. Along with the whole line trip and other hidden dangers, people's production and life has brought great impact. Some birds are not in nesting habitat in the tower, but the tower cross arm, the defecation will make the insulator pollution in the air, humidity, fog prone flashover accident.

Recent statistics show that, due to circuit fault caused by bird activity after lightning and external damage, has occupied third of the total number of bit line fault.

After field visits to the Sinopec Shengli Oilfield Co., Ltd. is the river oil production plant, for example, only a year in 2012 due to bird nesting caused by line outage 5 times, the impact of crude oil production of 55.1 tons.

According to the calculation of the market value of 3000 yuan per ton of crude oil, crude oil in 2012 due to bird caused by the loss of 165 thousand and 300 yuan. The cost of manpower and 
machinery for each outage maintenance is calculated by 20 thousand yuan, one year of 100 thousand yuan. The Sinopec of Shengli Oilfield Company Limited Xianhe oil production plant each year due to bird caused by the line loss of about 265 thousand and 300 yuan.

The national Power Grid Corp in December 17, 2004 issued a national grid [2004]641, the "notice" issued on the prevention of accidents and measures of power transmission equipment "national grid in forty-eight measures" in the anti bird work put forward specific requirements. To do a good job of transmission line maintenance work, improve the reliability of power grids, bird accident effective preventive measures, formulate feasible plan on the basis of bird activity rules, to prevent the bird near the tower, reducing the number of activity line due to failure of birds, to ensure safe and stable operation of power grid. At the same time, we have to do only anti bird and not to kill, not to hurt the birds, to promote the harmonious development of man and nature.

Effects of Birds on Orchards. It has become an urgent problem to solve the problem that the birds have been damaged. With the increase of the number of birds, the damage to the agriculture and fruit industry is also obvious. Harm birds on trees may be directly leaves, buds and fruit pecking, may also cause two damage on the basis of this, not only directly affect the quality and yield of fruit, but also lead to bacteria in the wounds were pecking fruit blooms, many normal fruit disease, severely affected the quality of fruit.

The Impact of Birds on the Airport. Bird strike event was defined as one of the major hazards of world class aviation crash. When birds fly in opposite directions, the relative speed between them is very fast, and it can also cause great damage. Data show that a weight of 500 grams of birds with a speed of about $370 \mathrm{~km}$ of the aircraft collided, will produce an impact of about 3 tons, equivalent to the aircraft was hit by a shell. The world has $20 \%$ plane crash accident is caused by bird strike aircraft, over the past 20 years, a total of 220 people died in a plane crash caused by bird strikes. Even if there is no cause of the crash, bird strike events may also cause flight delays, aircraft damage and other issues, the world's annual twenty thousand bird strikes occurred, causing economic losses of \$about 10000000000.

\section{Existing bird Repellent Products and Their Disadvantages}

There are many birds drive traditional currently on the market, including the red flag hanging and installation of bells, mirror, rotate the wind wheel, the bodies of birds in the farmland and orchard, put the scarecrow, inflatables and other physical methods, the method is simple, but for a long time, the birds will no longer fear them, unable to play the long-term effect of bird; bird of the chemical methods including spray bird repellents, even if the bird repellent uses green, also must use once every 15 days or so, is not universal and bird repellent effect is not significant.

In recent years, the market appeared some new anti bird device, the main use of sound, light, heat and other measures to stimulate shock or birds, so as to achieve the purpose of driving birds, such as bird thorn, radar bird, the bird driving device exist will cause problems, damage location monitoring is not accurate for birds. Therefore, the latest research and development of bird driving device is urgently needed to solve the problems such as the adaptability of birds, small range of birds, short effective time, high cost, no targeted blindness and so on.

\section{The Main Components and Functions of A New Bird Driving Product}

Selection of Bird Driving Mode. Birds of the natural enemies of the fear is inherent, not because of the long time the sound of the play makes the birds gradually adapt to lose the role of birds. Therefore, the device uses the way to play the voice of natural enemies to achieve the purpose of birds. In this part, only a suitable power of the speaker and a variety of natural enemies of the memory chip can be stored, the structure is simple and low cost.

Infrared Detection of Birds. In the choice of a good way to drive the birds, so that the speaker 24 hours a day has been working is not feasible, in the impact of the speaker's life, but also a great waste of electricity. Therefore, it is possible to use the passive pyroelectric infrared sensor BISS0001 as a device for sensing the birds. When there are birds close to the birds themselves issued by the infrared 
device is aware of the trigger to open the speaker, after a period of time, if the birds leave, the speaker will automatically shut down.

By access to information shows that the passive pyroelectric infrared sensor BISS0001 has the following characteristics: no need to use infrared or electromagnetic wave emission source, good concealment, mobile installation, high sensitivity, wide control range, with a pair of internal thermoelectric elements, a high resistance and a low noise FET, load No. 5 transistor (TO-5) shell. Its structure and equivalent circuit, as shown in the following figure.

In order to suppress the interference due to their temperature changes caused by the sensor is connected in series or differential balanced circuit in reverse thermoelectric element process, two characteristics consistent, thus able to detect non-contact infrared energy change object released, and convert it to electrical signal output. The pyroelectric infrared sensor to detect the biological form of non contact infrared radiation energy changes, and convert it into voltage signal, the amplified voltage signal, drive control circuit, infrared radiation detector will change from detected and received into the faint signal, the field effect in the probe the amplified output tube. Pyroelectric infrared sensor unit T1, T2, infrared feelings change in temperature sensitive unit, and the temperature change leads to the change of the electric signal, and the environment temperature change itself by its internal structure decides not to output signal, while the low frequency response of the sensor and the specific infrared wavelength (5 15 $\mathrm{m}$ response) determines the characteristics of the sensor on the outside of the infrared radiation caused by sensitive to temperature change itself, or only to birds or related to motion sensitive. Because of the detection signal voltage of pyroelectric infrared sensor output is very weak (usually only about $1 \mathrm{mV}$ ), and is a sign of change, the Finel lens effect and the output signal voltage is pulse (pulse voltage frequency is determined by the moving speed of the object to be measured, usually around $0.1 \sim 10 \mathrm{~Hz}$ ), in order to improve the detection the sensitivity of the detector to increase the detection distance, the general set up a Finel lens mounted on the front of the detector, and it can be matched with amplifier circuit, signal amplification to $120 \sim 150 \mathrm{~dB}$, which can be measured within 10 meters of the movements of birds.

Communication GPRS Module Design. The UB680 module integrates the GSM/GPRS wireless communication module and the GPS receiver module, both the GSM and GPS combination, GPS provides a variety of flexible working mode of information by the local control and transmission, can also control and air transport. 60 feet of the connector interface for, including: GSM power interface (Power Supply), GPS (Power Supply) power interface, ADC interface, GSM serial communication interface (2Channel UART), GPS serial communication interface (1 Channel UART), dual channel analog audio El (2 Channel Audio), $5 * 5$ matrix keyboard interface ( 5 x 5 Keypad Array), the standard SIM card interface, backup battery (RTC Backup). The module is embedded with the TCP/IP protocol to support the AT command expansion.

In the fusion mode, GPS positioning data through the GSM/GPRS unit and then transferred to the control board, the GPS data can be output in the serial port (mode 2), SMS transmission and GPRS transmission (working mode). To transmit the data to the standard NMEA - 0183 data format ("rdquo; at the beginning and end of $* \mathrm{cc}, \mathrm{CC} 2$ sixteen hexadecimal checksum), according to the different needs of customers, to parse the data into GPS easy to understand information interception. In this mode of transmission, control, serial output data, etc.. The AT module provides a series of instructions and extensible, convenient for users through the GSM_UARTO serial port of the GPS unit control and setting, to obtain the corresponding GPS positioning information, and through the GSM/GPRS unit of local or remote report back.

Signal Preprocessing Circuit Design. Pyroelectric infrared sensor dedicated control chip is used for receiving, amplifying, processing and control of sensor signal digital analog hybrid chip. The following figure is BISS0001 pyroelectric infrared detector discrete components of the circuit diagram. 


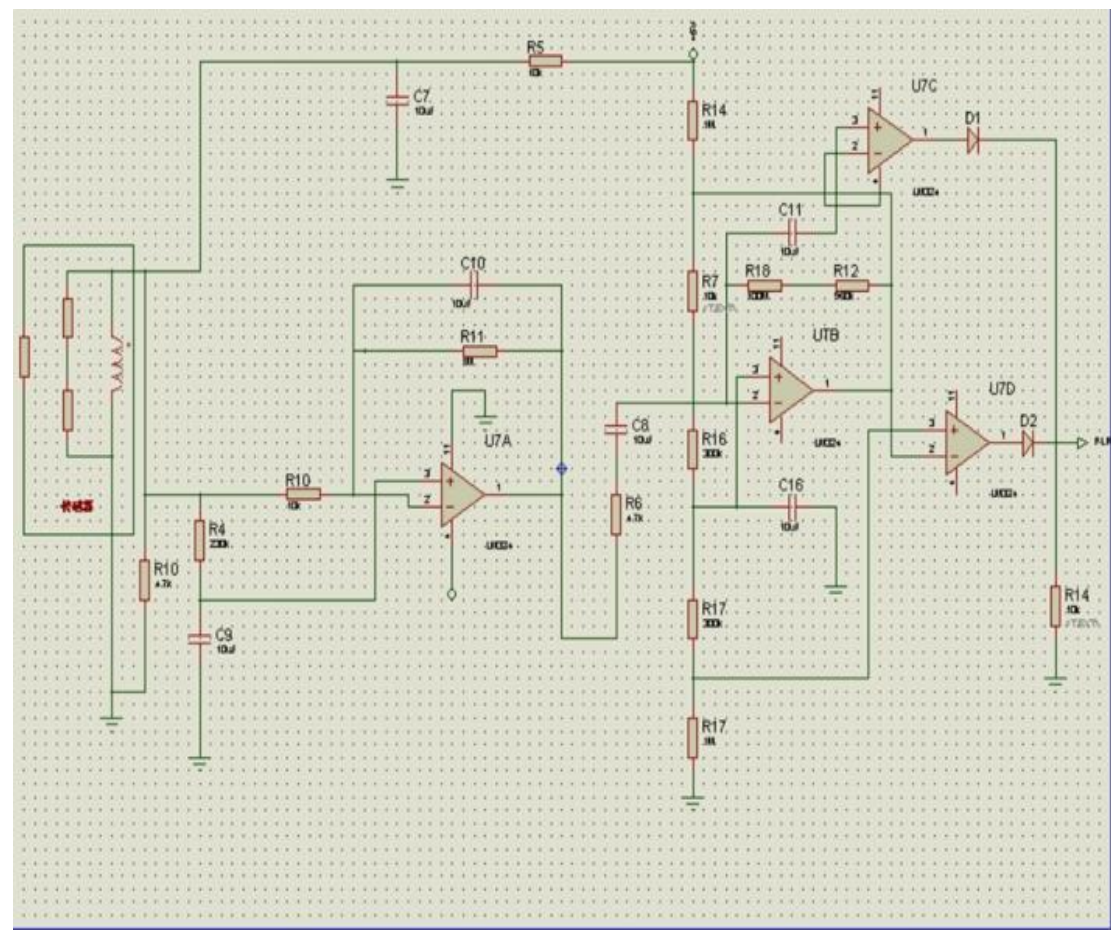

figure 1. BISS001 pyroelectric infrared detector discrete components internal circuit diagram

When the pyroelectric infrared sensor receives the infrared rays emitted by the object to be measured, the output of a low frequency weak signal to the BISS0001 chip to amplify the signal pretreatment, while the DC potential up to VM, the internal double amplitude discriminator detection trigger signal VS to start delay timer (as long as the trigger signal VS can jump along the start delay timer). Because $\mathrm{VH}$ is $0.7 \mathrm{VDD}, \mathrm{VL}$ is $0.3 \mathrm{VDD}$, so when $\mathrm{VDD}=5 \mathrm{~V}+1 \mathrm{~V}$ can effectively inhibit the noise interference, improve the reliability of the system. COP3 is a condition when the comparator input voltage $\mathrm{VC}<\mathrm{VR}(\mathrm{VR}=0.2 \mathrm{VDD})$, the $\mathrm{COP} 3$ output is low, shut the gate U2, trigger signal VS to the lower transfer; while VC>VR, COP3 output is high, then open the gate U2, enter the delay cycle. At this point, if there is a trigger signal VS jump arrival, start delay timer, while the VO pin output high level signal, access to sound and light alarm circuit will be able to achieve signal alarm.

Power Supply Circuit Design. The power supply circuit can realize the solar battery / battery switch. 2 diodes are used in the branch circuit of the battery, and when the two voltages are equal, the utility model is still powered by a solar panel. The method of the invention comprises an automatic charging circuit of a storage battery, a solar battery component, which can convert the solar energy into electric energy and supply the load to work or charge the battery pack. If the solar battery charging controller can be used to protect the battery charging and discharging, the battery pack is used for storing the electric energy and converting the direct current into alternating current. During the day, when the sun is shining, the solar battery supplies power to the device, while charging the battery. In the night or rainy day, when the solar battery module can not work, the battery pack is supplied to the load. 


\section{Product Appearance}

- Main control board

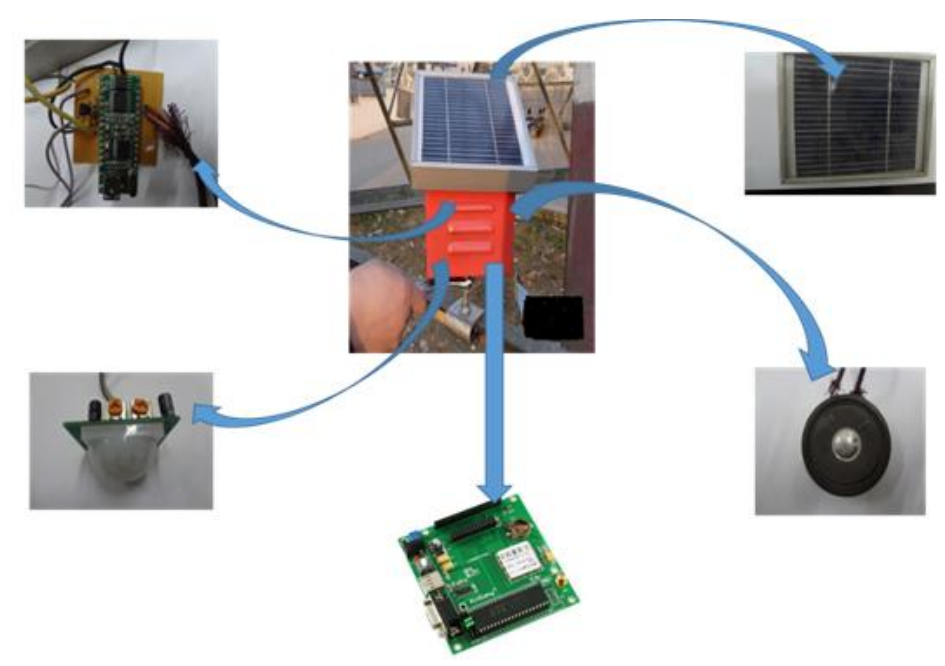

figure 2. Product appearance

The main control board is provided with a semiconductor memory chip, which can be connected with the computer through the USB interface, and then the call recording of the birds' natural enemies can be downloaded into the memory chip to store permanently. The main control board is embedded with an audio power amplifier circuit.

- Pyroelectric infrared sensor

The device is driven by a passive pyroelectric infrared sensor (BISS0001), and when the monitored object enters the infrared monitoring range, the infrared induction device moves, and the signal is transmitted to the main control board.

- Communication GPRS module

The main control board is connected with the communication GPRS module, and when the monitored object enters the monitoring range and continues to exist, it is possible to choose whether to notify the security personnel of the short message.

- speaker

The audio power amplifier circuit on the main control panel controls the loudspeaker and sends out the preset sound.

- Solar panel

Solar energy is used to collect the electric energy.

- FRP shell

The glass fiber reinforced plastic shell is made of synthetic silicone rubber as raw material, which is formed by high temperature vulcanization of HTV. The shell has the advantages of high flexibility, beautiful appearance, reliable insulation, corrosion resistance, aging resistance, long service life and rain proof.

\section{Product Operation and Testing}

Through the purchase of materials, manual welding circuit, the initial production of the product prototype - based on new energy and intelligence of the integrated monitoring of bird drive device. 


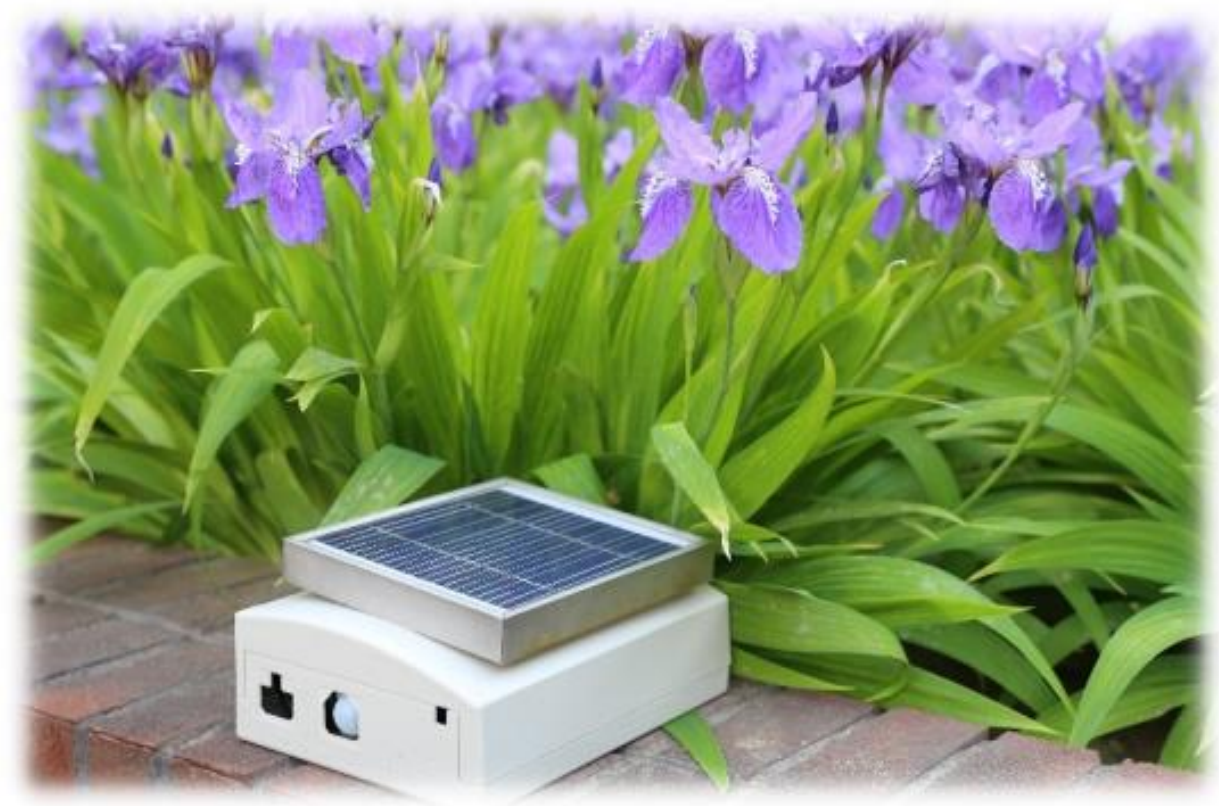

figure 3. Product physical map

Birds can feel the sound frequency range of $250 \sim 21000 \mathrm{~Hz}$, general hawk can make most of the birds have fear. With the destruction of the ecological environment, reducing the number of species, we first select the eagle as a built-in voice calls. Taking into account the field often windy weather, we will adjust the loudness of sound to $120 \sim 150 \mathrm{~dB}$, ensure no matter what operating environment (except for extreme weather such as storm, typhoon etc.), around ten meters, devices were able to work effectively. We can set the upper limit of loudness to 150 decibels due to a noise of about 165 decibels.
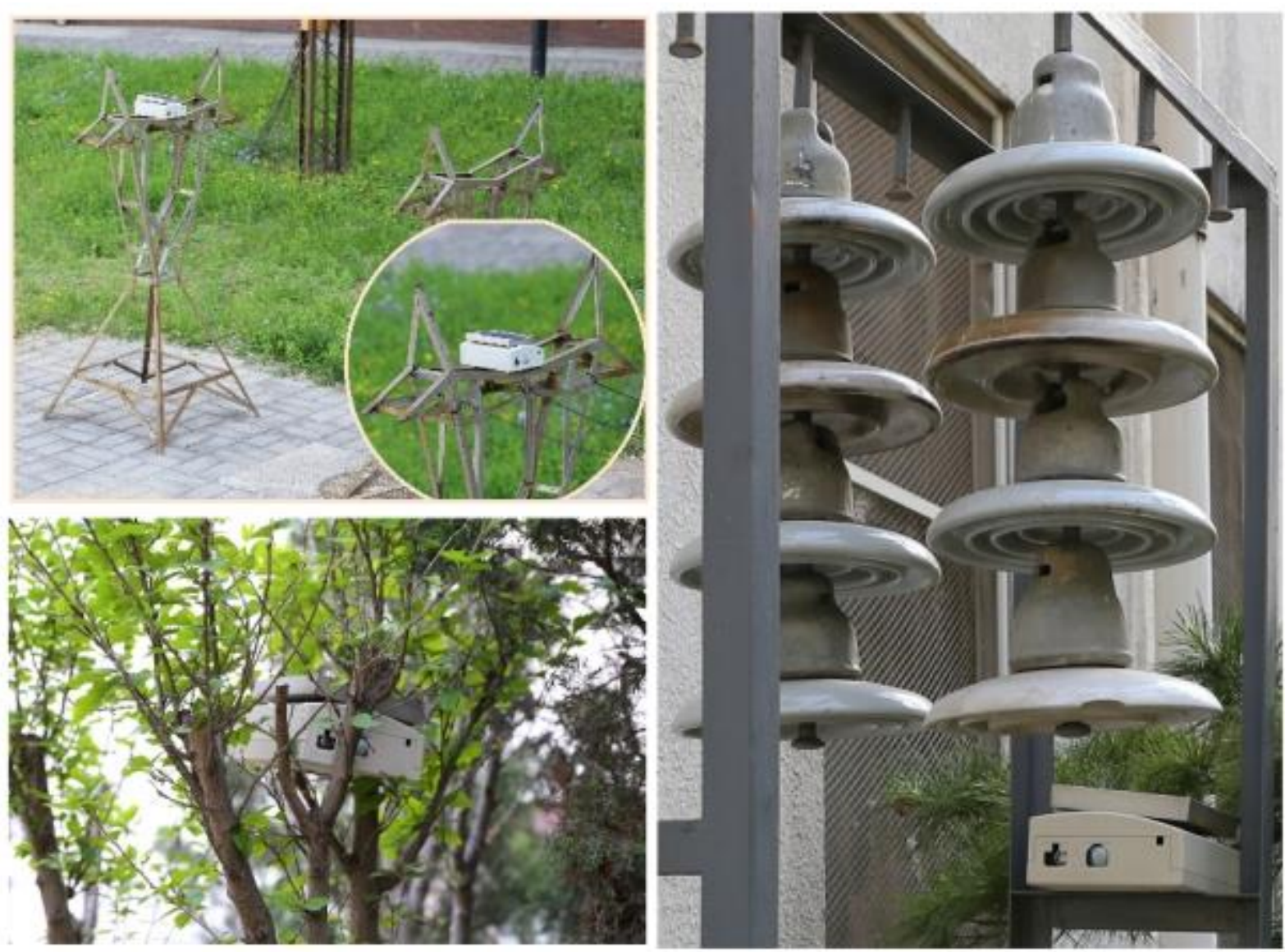

figure 4. Product run test scenario 
We test the product in the transmission line tower, insulator string, orchard, the test scene as shown in figure 29. The test results show that the device is sensitive and reliable in the actual test environment, and can realize the preset function.

\section{Product Innovation Point}

In the detection of harmful organisms, the use of their fear of natural enemies to call the warning, safe and environmentally friendly, no pollution, so the respect for life.

The speaker's voice can be downloaded according to the needs of the data line, the selection of large, strong applicability.

Through infrared sensor drive, fully automatic induction, intelligent control. The circuit has wide working range, large sensor sensing range and low power consumption.

Through the GPRS module to achieve the purpose of monitoring, can easily reflect the situation to the staff. The GPS receiver in the module can locate the specific location of the accident, which is convenient for the staff to check the line.

Low power consumption, accurate detection of the environment.

The light energy is converted into electric energy by the solar panel to supply the power for the system. Make full use of the new energy, to achieve the maintenance of the system.

\section{References}

[1] Z.J.Fan: The development of transmission line bird bird device research and drive. [D]. Inner Mongolia University .2006. [2](In Chinese)

[2] Y.Hui and J.L.Cui: Current status of transmission lines and lightning protection in China. [J]. High voltage technology .2001. (In Chinese)

[3] Y.X.Cao and D.X.Ding: Study on the bird proof device of the intelligent recognition and initiative driving [J]. Sichuan electric power technology .2013 (. Six ): Seventy-seven. (In Chinese)

[4] US Department of Transportation official website: www.transportation.gov/

[5] Anastasia D. Spiliopoulou; Ioannis Papamichail; and Markos Papageorgiou Toll Plaza Merging Traffic Control for Throughput Maximization, 10.1061/ ASCE 0733-947X 2010 136:1 67

[6] HUANG Juan-juan, ZHOU Zhou, LIN Xiang-sheng, Vehicle behavior analysis method and system, G08G1 / 01(In Chinese)

[7] Cassidy, M. J., and Rudjanakanoknad, J. 2005. "Increasing the capacityof an isolated merge by metering its on-ramp." Transp. Res., Part B:Methodol., 39, 896-913

[8] Keen, K. G., Schoffield, M. J., and Hay, G. C. 1986. "Ramp metering access control on M6 freeway." Proc., 2nd IEE Int. Conf. on Road Traffic Control, IEE, London.

[9] Kosmatopoulos, E. B., and Papageorgiou, M. 2003. "Stability analysis of the freeway ramp metering control strategy ALINEA." 11 th IEEE Mediterranean Conf. on Control and Automation CD-ROM.

[10]Papageorgiou, M., Hadj-Salem, H., and Blosseville, J. M. 1991. “ALINEA: A local feedback control law for on-ramp metering." Transportation Research Record. 1320, Transportation Research Board, Washington, D.C., 58-64 